\title{
LA REFORMA AGRARIA CHILENA: ENTRE EL GRADUALISMO Y LA TRANSFORMACIÓN ACELERADA
}

Octavio Avendaño Pavez 


\section{OCTAVIO AVENDAÑO PAVEZ}

Octavio Avendaño Pavez es Doctor en Ciencia Política, Universidad de Florencia, Italia; Sociólogo de la Universidad de Chile y Profesor de Historia de la Universidad de Concepción. Es académico del Departamento de Sociología de la Universidad de Chile y del Departamento de Ciencia Política y Relaciones Internacionales de la Universidad Alberto Hurtado. Sus áreas de investigación se han orientado al análisis del cambio institucional, los grupos de interés, el desarrollo histórico de la representación y la política comparada. Es autor del libro Los partidos frente a la cuestión agraria en Chile, 19461973. Representación de intereses, gradualismo y cambio estructural (Santiago: Lom Ediciones, 2017). Coautor del libro La hacienda revivida. Democracia y ciudadanía en el Chile de la transición (Santiago: RIL, 2003) y de Sociología. Introducción a los clásicos (Santiago: Lom Ediciones, 2012). También es autor de varios capítulos y artículos publicados en revistas nacionales y extranjeras. 


\section{LA REFORMA AGRARIA CHILENA: ENTRE EL GRADUALISMO Y LA TRANSFORMACIÓN ACELERADA}

\section{INTRODUCCIÓN}

La Reforma Agraria ha sido, sin duda, el proceso de transformación estructural más importante que se ha llevado a cabo desde los inicios de la vida republicana. Junto a la experiencia peruana, la Reforma Agraria chilena destacó por haber llevado a cabo políticas destinadas a modificar la gran propiedad, distribución territorial, e incorporación campesina. En tal sentido, se trata de un proceso que destaca, de manera significativa, en comparación al resto de las reformas agrarias impulsadas en el continente a partir de la política de Alianza para el Progreso, que se inicia con la conferencia de Punta del Este celebrada en 1961. En lo que respecta a la experiencia chilena existen una serie de antecedentes que permiten plantear que la Reforma Agraria obedeció a factores endógenos, asociados a la situación de postergación y déficit productivo que venía registrando la agricultura desde la década de 1930. Dentro de estos factores sobresalen también las propuestas que los partidos de izquierda, así como los sectores socialcristianos, venían planteando desde que se configuran e intervienen en la discusión pública en torno a esa misma época.

Por otra parte, los factores endógenos también se pueden reconocer a partir de los cambios en la representación política que provocan las reformas institucionales y en la normativa electoral, aprobadas en 1958. De ellas, la más relevante fue el establecimiento de la "cédula única", que puso fin al cohecho y al control electoral que ejercía la derecha en las zonas rurales (Lapp. 2004; Gamboa, 2011). Esto permitió el posicionamiento de aquellos partidos que venían promoviendo cambios estructurales en el sector rural, cuyos planteamientos se complementaron con los diagnósticos y propuestas que surgieron, en el contexto de los años sesenta, de diversos organismos internacionales, entre ellos la Comisión Económica para América Latina (Cepal), la Organización de Naciones Unidas para la Alimención y la Agricultura (FAO), y el Comité Interamericano de Desarrollo Agrícola (CIDA).

La implementación de la Reforma Agraria chilena se fue dando de manera gradual, desde que se aprueba la primera legislación en 1962, bajo el gobierno de Jorge Alessandri (1958-1964). Como es sabido, la reforma agraria de Alessandri tuvo un carácter marcadamente limitado, que se puede incluso comparar a las políticas de colonización que se habían impulsado previamente en Chile, o a iniciativas similares 
en otros países de la región (Frank, 1965; García, 1967; McCoy, 1969; Chonchol, 1971, 1994). Pese al carácter limitado que tuvo la reforma, tal como se verá en este artículo, Alessandri logró establecer buena parte de la institucionalidad a través de la cual se organiza el proceso de expropiación y distribución territorial, en conjunto a la organización campesina, en los dos gobiernos sucesivos de Eduardo Frei Montalva (1964-1970) y el de Salvador Allende G. (1970-1973). Con la aprobación de la ley 16.625 de Sindicalización Campesina y la ley 16.640 de Reforma Agraria, ambas durante el primer semestre de 1967, se da inicio a una fase de transformación que tendrá un efecto irreversible (Gómez y Echenique, 1988). Con la aprobación de ambas leyes comenzaba una fase de expropiación del latifundio improductivo, organización del sector reformado y fomento de la sindicalización campesina (Barraclough y Fernández, 1974; Cereceda y Dahse, 1980; Huerta, 1989; Moreno, 2013; Bengoa, 2015). A su vez, se trata de una fase de mayor participación y democratización de la sociedad chilena.

A pesar de estos logros, en el transcurso de las dos últimas décadas es posible reconocer una serie de opiniones críticas de lo que fue dicha experiencia. Una de las críticas estuvo orientada a poner el acento en el carácter supuestamente excesivo y discriminatorio con los terratenientes que habría tenido el proceso de expropiación de las grandes haciendas (Cousiño y Ovalle, 2013), reafirmando con ello lo que con anterioridad habían destacado autores vinculados a la derecha chilena (Cardemil, 2000; Fontaine A., 2001). En la misma línea, otros autores han planteado que, desde el punto de vista productivo, la experiencia de la Reforma Agraria habría sido negativa. Por el contrario, el aumento de la productividad habría sido consecuencia de "la privatización, la desregulación y el establecimiento de los derechos de propiedad sobre la tierra" (Valdés y Foster, 2015: 85). En lo que respecta al fenómeno de las expropiaciones, la ley 16.640 fue bastante clara en señalar que dichas medidas se aplicaban a predios mal trabajados y explotados de manera deficiente, cuestión que se mantuvo durante el gobierno de la Unidad Popular (1970-1973). Si bien hubo sectores de la izquierda que se mostraron partidarios de incluir a todos los terratenientes sin excepción, esa disposición no fue manifestada ni por el gobierno de Frei Montalva ni por el de Allende. Por otra parte, balances sobre el aumento de la producción durante el periodo comprendido entre 1967 y 1973 han sido reconocidos por diversos estudios e investigaciones que se han venido realizando, tanto en el medio nacional (Gómez y Echenique, 1988; Cepal, 1986) como en el extranjero (Tinsman, 2016). Esos estudios coinciden en destacar que durante el periodo de implementación de la Reforma Agraria se registran aumentos, aunque leves, en la producción de rubros tradicionales, pero un incremento considerable de la producción frutícola y luego forestal, como consecuencia del plan de desarrollo que la Corfo impulsó desde la segunda mitad de los años sesenta. Sin la transformación 
estructural de la Reforma Agraria y sin el Plan de Desarrollo Frutícola y Forestal que la Corfo implementa en paralelo, difícilmente se hubiese llevado a cabo un desarrollo agroexportador como el que se conoce en el periodo sucesivo.

El propósito de este artículo es analizar la implementación de la Reforma Agraria en Chile, haciendo hincapié en la fase de implementación de la ley 16.640, aprobada en junio de 1967. Se pone atención en el comportamiento de las organizaciones que promovieron la expropiación de las grandes haciendas y la sindicalización campesina en la fase de implementación del proceso reformista. A medida que se avanza en el proceso de Reforma Agraria se acentúa la distancia entre los partidos que impulsan la aceleración de las expropiaciones con miras a debilitar a los terratenientes, respecto de quienes apuestan por una transformación gradual, respetando la institucionalidad y la legalidad vigente.

\section{LAS PARTICULARIDADES DE LA REFORMA AGRARIA CHILENA EN PERSPECTIVA COMPARADA}

En Chile, la Reforma Agraria fue asumida de manera gradual, como si hubiese sido parte de un proceso evolutivo. Se inicia con la aprobación de la ley 15.020, en 1962, durante el gobierno de Jorge Alessandri (1958-1964). Se hace efectiva durante el gobierno de Frei, quien se avala en los primeros años de la legislación y la institucionalidad creada durante el gobierno de Alessandri, mientras se aprueba una nueva ley de Reforma Agraria, 16.640. Esta legislación, ratificada en junio de 1967, unida a una reforma constitucional previa, permite llevar a cabo la expropiación de predios en manos de privados. La ley 16.640 se mantuvo durante todo el gobierno de la Unidad Popular, al igual que los organismos inicialmente creados bajo el gobierno de Alessandri.

De acuerdo a lo que se planteaba en el texto original de la ley 15.020, aprobada en 1962, el propósito de la Reforma Agraria debía ser facilitar el acceso a la tierra y contribuir a aumentar la producción agropecuaria. Con dicha normativa, la antigua Caja de Colonización Agrícola se transformaba en la Corporación de la Reforma Agraria (CORA), la que se encargaría de la administración de todos aquellos predios que fueran expropiados por razones de abandono o porque estuvieran mal trabajados. Junto a la CORA se crea el Instituto de Desarrollo Agropecuario (Indap), encargado de asesorar y entregar asistencia técnica a los pequeños y medianos productores. El Indap también debía promover la formación de cooperativas agrícolas. Ambas instituciones se mantuvieron todo el periodo comprendido entre 1962 y 1973. Durante el gobierno de Eduardo Frei las funciones y recursos de ambos organismos aumentaron considerablemente. Del mismo modo, sus líneas de 
acción estuvieron orientadas a la gestión del sector reformado y al fomento de la organización campesina.

Pero el gobierno de Alessandri no llevó a cabo una política de redistribución que beneficiara al segmento de la población campesina que carecía de tierra (Garrido, et al, 1988; Huerta, 1989; Bengoa, 2015). Por el contario, se trató de una reforma que se remitió, principalmente, a fomentar la colonización de terrenos fiscales, algunos de los cuales fueron adquiridos como consecuencia del abandono y la deficiente producción que registraban hasta el momento de la venta. Tampoco se llevó a cabo una política de integración del campesinado, dado que las familias beneficiarias no necesariamente poseían ese origen social. Pese a estas limitaciones, la legislación aprobada bajo Alessandri fue clara en la necesidad de expropiar aquellos predios que estuvieran mal trabajados o que hubiesen sido explotados de manera ineficiente. Adicionalmente, un año después de la aprobación de la ley 15.020, el gobierno de Alessandri promovió una reforma constitucional a través de la cual se autorizó el pago en diferido de las indemnizaciones de los predios que resultaran expropiados por razones de utilidad pública. De manera que el gobierno de Alessandri proporcionó una base institucional y jurídica que terminó siendo ampliada y perfeccionada durante el sucesivo gobierno de Eduardo Frei Montalva (1964-1970).

Si se compara la experiencia chilena con la de otros casos latinoamericanos, el carácter efectivo de la Reforma Agraria se adquiere antes de la aprobación de la ley 16.640, debido a que desde el inicio del gobierno de Frei se plantea la expropiación de predios en manos de privados y la distribución territorial en aquellos adquiridos por el Estado. De hecho, la constitución de asentamientos se comenzó a implementar pocos meses después de asumido el gobierno. Los proyectos de Sindicalización Campesina y de Reforma Agraria enviados por el Ejecutivo a fines de 1965 serán aprobados en abril y junio de 1967, respectivamente. En el intertanto, Frei debió operar con la legislación y la institucionalidad establecida bajo el gobierno de Alessandri (Moreno 2013; Avendaño, 2016). La aprobación de la ley 16.640, claro está, permitió aumentar el número de expropiaciones junto con integrar a una parte de los campesinos a participar del sector reformado. La legislación sobre Reforma Agraria y Sindicalización Campesina se mantiene durante el gobierno de la Unidad Popular (1970-1973). En este gobierno la Reforma Agraria entra en una fase de mayor profundización y aceleración. Aun así se van a producir tensiones al interior de la coalición de gobierno, que se verán reforzadas con las críticas de la derecha y la oposición de los grandes terratenientes. 
Tabla 1. Algunos intentos de tipificación de las reformas agrarias en América Latina

\begin{tabular}{l|l|l} 
Tipo de reforma & Modo de implementación & Ejemplos y casos \\
\hline Colonización & $\begin{array}{l}\text { Ocupación de tierras fiscales } \\
\text { asignadas por el Estado }\end{array}$ & $\begin{array}{l}\text { Bolivia, desde 1937 } \\
\text { Chile, 1929-1966 } \\
\text { Ecuador, 1930-1996 } \\
\text { Honduras, 1952-1990 }\end{array}$ \\
\hline Reforma productivista & $\begin{array}{l}\text { Sin expropiación, énfasis en } \\
\text { la producción y ocupación de } \\
\text { terrenos fiscales }\end{array}$ & $\begin{array}{l}\text { Chile, 1962-1964 } \\
\text { Colombia, 1958 } \\
\text { Venezuela, 1958 }\end{array}$ \\
\hline Expropiación y distribución & $\begin{array}{l}\text { Expropiación a grandes } \\
\text { terratenientes e integración } \\
\text { campesina }\end{array}$ & $\begin{array}{l}\text { Guatemala, 1954 } \\
\text { Perú, 1964 } \\
\text { Chile, 1967-1973 }\end{array}$ \\
\hline Expropiación y distribución & $\begin{array}{l}\text { Expropiación y cambio en } \\
\text { la estructura de poder bajo } \\
\text { experiencias revolucionarias }\end{array}$ & $\begin{array}{l}\text { México, 1917 en adelante } \\
\text { Bolivia, 1953-1954 } \\
\text { Cuba, 1959-1966 } \\
\text { Perú, 1969-1976 }\end{array}$ \\
\hline
\end{tabular}

FUENTE: Elaboración propia a partir de García (1967); McCoy (1969: 9); Chonchol (1994); Albertus (2015: 126).

En la bibliografía especializada se advierte un intento de tipificación mediante la cual es posible identificar tres experiencias de reforma agraria distintas entre sí, en sus aspectos procedimentales y en relación a sus objetivos (Frank, 1965: 184ss.; Mattelart, 1972: 72ss.; Chonchol, 1971: 270). El primer tipo de reforma agraria correspondería a aquellas que fueron impulsadas por las oligarquías y los propios terratenientes. El énfasis estuvo puesto en elevar los niveles de producción y en tecnificar muchas de las faenas que se llevaban a cabo al interior de las grandes propiedades. Sin embargo, este tipo de reformas no implicó la modificación del régimen de propiedad ni tampoco la condición que poseían los grandes terratenientes ${ }^{1}$. El segundo tipo de reforma corresponde a aquellas que integran a los campesinos y distribuyen la tierra entre quienes resultan beneficiarios. En este tipo de reforma se lleva a cabo la modificación de la gran propiedad, pero respetando la legalidad vigente. El tercer tipo de reforma, en cambio, es el que se impulsa en el marco de procesos revolucionarios, en los cuales se modifica por completo la estructura productiva y las relaciones de poder vigentes hasta ese momento.

1 En opinión del sociólogo colombiano Antonio García, este tipo de reforma agraria, que él denominaba "convencional", "no se orientó hacia la remoción de los obstáculos estructurales que impiden o frenan el desarrollo, subsidiando la estructura tradicional por medio de una expansión del mercado de tierras, tutelada por el Estado" (García, 1967: 22). 
El primer tipo de reforma agraria se habría producido en el marco de gobiernos conservadores, como los de Venezuela y Colombia a fines de los años cincuenta. El segundo tipo se impulsó en el marco de gobiernos reformistas, como el de Lázaro Cárdenas en México, o de Eduardo Frei en Chile. En el tercero la experiencia paradigmática fue la de la Revolución Cubana. Esta tipología se mantuvo durante todo el periodo de vigencia de la política de Alianza para el Progreso. Incluso, en el tercer tipo se extendió hasta la Revolución Nicaragüense iniciada en 1978. En el último tiempo la bibliografía especializada añade un cuarto tipo de reforma, que corresponde a las que se aplican en el marco de regímenes autocráticos (Albertus, 2014). Un buen ejemplo de este cuarto tipo sería la reforma agraria implementada por el régimen militar peruano, encabezado por el general Juan Velasco Alvarado entre 1969 y 1976.

A partir de la tipología anterior es posible sostener que en Chile se registraron las tres primeras reformas mencionadas. En efecto, el primer tipo de reforma fue asumido por el gobierno de Jorge Alessandri, dado que lo implementado en ese mandato se remitió más bien a una política de colonización en terrenos fiscales, sin que se hubiese alterado el régimen de propiedad privada. Como ya hemos dicho, los mayores avances que se produjeron en este gobierno se expresaron en una institucionalidad que será utilizada posteriormente y en la reforma constitucional que autoriza al Estado el pago en diferido por efecto de las expropiaciones. El segundo tipo, e incluso el tercero se habrían registrado en el gobierno de Frei Montalva. De hecho, la profundización del proceso de reforma, que se hizo efectivo a partir de 1970, fue el resultado de diversas iniciativas legales aprobadas en los años 1966, 1967 y 1969. Cabe agregar, además, que la Reforma Agraria tuvo un carácter irreversible. Ni siquiera con la llamada "contrarreforma", que los militares aplicaron a partir de 1974, fue posible restablecer la situación que existía en el agro chileno antes de 1964.

\section{DE POLÍTICA DE COLONIZACIÓN A REFORMA AGRARIA EFECTIVA}

En los años previos a la aprobación de la primera ley de Reforma Agraria, en 1962, venían siendo difundidos una serie de diagnósticos sobre la situación deficitaria que la agricultura arrastraba desde, al menos, los inicios de la década de 1930. En ellos se insistía en la incapacidad que tenía la producción agrícola para abastecer la demanda de alimentos manifestada por la población urbana. Por ende, los gobiernos que se sucedieron hasta antes de 1964 se vieron en la necesidad de destinar importantes sumas a la inversión de productos agrícolas y pecuarios. Los diagnósticos difundidos también hacían hincapié en la condición de los campesinos minifundistas, cuyas 
economías no lograban asegurar la sobrevivencia; la precariedad de los trabajadores asalariados y el problema de la concentración territorial. A ello se agregaba la disminución de las superficies de riego para uso agrícola como consecuencia de la concentración del recurso hídrico, que generó la concesión de derechos a privados desde que fuera promulgado el Código de Aguas de 1951.

Para revertir la situación deficitaria de la agricultura y al mismo tiempo los problemas de acceso a la tierra de parte de la población minifundista, algunos de los diagnósticos más relevantes que surgieron en el medio nacional, como los de Aníbal Pinto (1958) y Jorge Ahumada ([1958] 1990), propusieron que fuera fomentada la creación de una clase media rural a través de medidas de tipo distributivas. La creación de una clase media rural tendría, a su vez, otro importante efecto, ya que podría servir de soporte para evitar situaciones de conflictividad social. Este tipo de planteamiento fue complementado con el diagnóstico y las propuestas que surgieron de los organismos internacionales dedicados a analizar el problema agrario en la región, que se manifestaron a favor de la Reforma Agraria, y de otras medidas de distribución territorial que permitieran ampliar el mercado interno al elevar el poder adquisitivo de la población rural, especialmente aquella de origen campesino.

A inicios de los años sesenta, mientras transcurría el gobierno de Jorge Alessandri (1958-1964), se hizo inminente la implementación de la Reforma Agraria. Como se reconoce en un libro que asume una postura más bien reticente frente al proceso reformista en general: "Para esta fecha, ya quedaba muy claro la necesidad imperiosa de llevar a la práctica un proyecto de reforma agraria chilena y luego concretarlo a través de una ley" (Garrido, et al, 1988: 85). Varios fueron los factores que se conjugaron a favor de dicha implementación. En primer lugar, el debilitamiento experimentado por la derecha luego de las elecciones de 1961 y los efectos que había generado, en desmedro de ese sector, el establecimiento de la "cédula única" en 1958. En segundo lugar, el ingreso de representantes del Partido Radical (PR) al gobierno, ocurrido poco después de las elecciones parlamentarias de 1961. Los radicales habían formulado una propuesta a favor de la Reforma Agraria en la XXI Convención de ese partido, efectuada del 22 al 25 de junio de 1961 (Gómez, 2003). Una vez en el gobierno decidieron impulsar esa reforma con la finalidad de neutralizar la influencia que estaban alcanzando, en ese entonces, los partidos integrantes del Frente de Acción Popular (FRAP), el Partido Comunista (PC) y el Partido Socialista (PS) (Avendaño, 2017: 227). En tercer lugar, el impulso que se dio a las reformas agrarias con la política de Alianza para el Progreso, promovida por el presidente Kennedy en el marco de la conferencia de Punta del Este realizada 
en 1961². Según recuerda Chonchol (1994: 266), la Alianza para el Progreso ligaba la ayuda financiera de Estados Unidos a un compromiso de efectuar cambios estructurales, entre los que se planteaba la realización de la Reforma Agraria y la reforma tributaria.

Cabe destacar, a modo de comentario general, que en Chile, a diferencia de lo que ocurría en otros países como por ejemplo Perú, no existía una presión campesina por la distribución territorial ${ }^{3}$. Tampoco se había logrado configurar un movimiento campesino con acciones y formas de organización distribuidas en las principales provincias de la zona centro y sur del país, con excepción de experiencias aisladas y la influencia que lograban ejercer instituciones ligadas a la Iglesia, o bien grupos falangistas y comunistas (Affonso, et al, 1970; Loveman, 1976; Bruna, 1985). En parte, ello se debía a las restricciones que en Chile existían desde 1947 con la ley 8.811 impulsada por el gobierno de González Videla (1946-1952), que limitaba la organización de sindicatos campesinos. En parte, también, por la vigencia de cerca de diez años de la Ley de Defensa de la Democracia, establecida bajo ese mismo gobierno en 1948, que había proscrito al PC y prohibido la agitación social en las principales áreas productivas. Las limitaciones mencionadas contribuyeron a mantener las relaciones de dominación y de sumisión que se habían arraigado en los siglos XVIII y XIX. Recién a partir de 1958 se observa una mayor preocupación de los partidos por atraer el voto campesino y promover la organización de sindicatos y federaciones provinciales. Para tal propósito resultaron gravitantes las reformas políticas introducidas en 1958.

Alessandri envió un proyecto que concitó la crítica tanto de parlamentarios del FRAP como de representantes del Partido Demócrata Cristiano (PDC). Fueron representantes del Partido Radical (PR) quienes redactaron el proyecto de ley enviado por el Ejecutivo (Huerta, 1989: 134). Apenas se dio inicio a la discusión se puso en el tapete el problema de la propiedad. El hecho de plantear la Reforma Agraria como tema de agenda implicaba debatir sobre la necesidad de mantener o bien modificar el artículo 10 de la Constitución de 1925. Los parlamentarios de la derecha

2 En lo inmediato, la reforma agraria promovida por la Alianza para el Progreso en países como Colombia, Venezuela y Perú a inicios de los años sesenta se tradujo en una política de redistribución bastante limitada, dado que la subdivisión o parcelación de los latifundios no tuvo mayor relevancia desde el punto de vista del desarrollo económico. De hecho, por lo general, la subdivisión y parcelación implicó la entrega de tierras poco fértiles o de menor calidad (García, 1967: 22; Chonchol, 1994: 279ss.).

3 Como bien describe Bengoa, en el campesinado chileno "formado por inquilinos, 'obligados', voluntarios, afuerinos, peones de a pie, medieros, mujeres lecheras, niños yunteros, no estaba puesta la disyuntiva de deshacerse de los patrones por vías racionales” (Bengoa, 2016: 56). 
consideraban que la Reforma Agraria debía servir para estimular la producción, para lo cual bastaba con tecnificar las faenas y facilitar la inversión tecnológica en los grandes predios ${ }^{4}$. Asimismo, consideraban que la Reforma Agraria debía seguir en la misma línea de la Caja de Colonización Agrícola, esto es, ser instrumento orientado a la "privatización de las tierras pertenecientes al Estado" (Cf. Avendaño, 2017: 229). Por ende, según recuerda Huerta (1989: 136), los representantes de la derecha, liberales y conservadores consideraron necesario emular la reforma agraria que desde 1959 venía llevando a cabo el gobierno venezolano encabezado por Rómulo Batancourt, perteneciente a la Acción Democrática.

De parte de la principal organización de los terratenientes, la Sociedad Nacional de Agricultura (SNA), inicialmente se aceptó la propuesta de una Reforma Agraria cuyo énfasis estuviera puesto en la producción y no en la modificación de la gran propiedad. Incluso, dentro de la propia SNA hubo sectores que se mostraron dispuestos a respaldar iniciativas de subdivisión de haciendas improductivas. Después de todo, existían miembros de esa organización gremial que también estaban vinculados a la producción industrial (Urzúa, 1973; Avendaño y Escudero, 2016). A eso se agrega el hecho de que para la SNA, a inicios de los sesenta y tomando en cuenta las condiciones de la época, concitaba menos preocupación la Reforma Agraria que, en cambio, la sindicalización campesina (Oszlak, 2016: 119120).

Mientras Alessandri se aprontaba a enviar el proyecto al Congreso Nacional, la Iglesia Católica decidió llevar a cabo un proceso de asignanción de tierra a campesinos tras la subdivisión de predios de su propiedad en el Obispado de Talca y en el Arzobispado de Santiago. Se estima que entre ambas zonas fueron subdivididas 5.545 hectáreas, el $90 \%$ de riego, entre 200 familias que residían y se desempeñaban en los predios de la Iglesia (Huerta, 1989: 144). La Iglesia se manifestó favorable a una reforma agraria que implicara redistribución de tierra a trabajadores agrícolas y población dependiente del minifundio. La Conferencia Episcopal emitió una declaración en donde daba a conocer la posición de la Iglesia a favor de una reforma agraria efectiva, que implicara la expropiación, subdivisión de tierra y su posterior distribución entre la población campesina. En ella se decía: "Las condiciones en que se encuentra en nuestros días el sector campesino, como hemos visto, reclaman con urgencia una profunda transformación de la estrctura rural" (Pastoral Colectiva del Episcopado de Chile, 1962: 20). En seguida, la declaración

4 En opinión de Sofía Correa, al aceptar de ese modo la Reforma Agraria, "los partidos de la derecha escogían el camino de la negociación, descartando el de la resistencia al cambio" (Correa, 2004: 240). 
emitida por la Iglesia advertía que la Reforma Agraria no podría ser concebida sin los "dolorosos sacrificios que ciertas restricciones impondrán a muchos que hasta el presente han sido indudablemente privilegiados en cuanto a su situación social y económica" (Ibid:: 24).

La Reforma Agraria de Alessandri tuvo un impacto bastante limitado si se compara con lo que va a ocurrir en los dos gobiernos posteriores, el de Frei Montalva y de Salvador Allende. En la práctica, la CORA, que junto con el Indap se establecieron con la ley 15.020 de Reforma Agraria, no aplicó sus facultades expropiatorias a predios que tenían la condición de haberlo sido por el hecho de encontrarse mal explotados. Fue por eso que políticos de izquierda apodaron la Reforma Agraria de Alessandri con el nombre de "reforma de macetero". Pese a todo, como se ha reiterado en este artículo, con la creación de la CORA y el Indap se logró establecer la institucionalidad en base a la cual se organizaría el proceso de expropiación, organización e incorporación de los campesinos durante los dos gobiernos sucesivos. Del mismo modo, al año siguiente, en 1963, se logró aprobar una importante reforma constitucional que le otorgaba al Estado la posibilidad de realizar pagos en diferido, con un máximo de quince años, por efecto de expropiación. Como explicó el mismo Alessandri en su mensaje del 21 de mayo de 1964, el propósito de este importante cambio constitucional era permitir "el pago en diferido en las expropiaciones que se realicen para llevar a efecto la reforma agraria, legislación que está llamada a facilitar el cumplimiento de las finalidades tenidas en vista para modificar el sistema de tenencia de la tierra" (Cf. Goic y Sanhueza, 1992: 45). De manera que fue en el marco del gobierno de Alessandri que se vio alterado un aspecto que la derecha había tendido a sacralizar, como ocurre con la defensa irrestricta del derecho de propiedad (Brahm, 1999: 172ss.; Moreno, 2013: 52).

Tomando en cuenta lo que fue el debate y el proceso legislativo, la Reforma Agraria propuesta por Alessandri no concitó el mismo respaldo que sí tuvo la reforma constitucional aprobada en 1963. En el intertanto, los partidos del FRAP y del PDC intensificaron sus acciones en localidades rurales y al interior de grandes predios a fin de movilizar y organizar al campesinado (Affonso, et al, 1970; Loveman, 1976). Además lograron precisar y definir los principales temas que debía incluir el proyecto de Reforma Agraria. Pese al discurso antioligárquico que sobresalía entre los dirigentes del FRAP, pertenecientes al PC y al PS, en lo sustantivo había coincidencia con el PDC respecto a la necesidad de eliminar el latifundio improductivo, revertir los déficit que registraba la producción agropecuaria, facilitar el acceso a la tierra de la población campesina y fomentar su organización en sindicatos y cooperativas. Pero a diferencia de los partidos del FRAP, el PDC sería capaz de elaborar la propuesta que haría más viable, desde el punto de vista jurídico, una reforma que implicara la expropiación de predios privados (Avendaño, 2016, 
2017). Para tal finalidad, juristas y exponentes de la vertiente "comunitarista" dentro del PDC invertirían aquel argumento que se utilizaba para la defensa del derecho de propiedad. Es decir, si para la defensa irrestricta de la propiedad privada se aludía al derecho natural, a la misma fuente se hacía referencia para justificar la distribución de la tierra a quienes habiéndola trabajado no podían acceder a ella.

\section{HACIA UNA DEMOCRACIA EFECTIVA}

Tras el triunfo obtenido en las elecciones del 4 de septiembre de 1964, Frei Montalva se abocó a organizar lo que sería su gobierno y a definir los equipos de trabajo que se encargarían de asumir las principales funciones en el Ministerio de Agricultura, en la CORA y en el Indap. Desde que se da inicio al nuevo gobierno, la CORA y el Indap, que habían sido creados con la ley 15.020, fueron aumentando sus atribuciones, recursos y número de funcionarios (Moreno, 2013: 98ss.). Y aumentaron mucho más luego de que en junio de 1967 se aprobara la ley 16.640 de Reforma Agraria. Al inicio de su gobierno, Frei además se dedicó a formar otras comisiones destinadas a la elaboración de los proyectos de sindicalización campesina y de Reforma Agraria, así como de formular una propuesta destinada a modificar el artículo 10 de la Constitución de 1925, que garantizaba el derecho y la defensa de la propiedad.

El proyecto de Reforma Agraria formulado y enviado a la discusión en el Congreso Nacional en noviembre de 1965 contemplaba la expropiación de todos aquellos predios que estuvieran mal trabajados y explotados que sobrepasaran las 80 hectáreas de riego básico (HRB). Bajo ninguna circunstancia se contemplaba la expropiación de aquellas unidades que producían de manera adecuada, así como tampoco las superficies en zonas cordilleranas o en el secano costero. El proyecto además contemplaba que los predios expropiados pasaban a ser organizados mediante la modalidad de asentamientos. Los asentamientos fueron concebidos como formas de propiedad colectiva de carácter transitoria, que luego de cinco años permitían a los beneficiarios optar por mantener esa modalidad o bien por la parcelación y asignación individual a cada familia. Adicionalmente, por medio del sistema de asentamientos se intentaba estimular la participación de los campesinos en la gestión y en la posterior distribución de los beneficios de la explotación (Echenique, 1972: 104ss.). Desde luego, también existía un criterio de racionalización de los recursos al organizar de manera colectiva el trabajo y la producción. El sistema de asentamientos fue establecido antes de que se aprobara la nueva ley de Reforma Agraria, elaborada y enviada por el gobierno. De hecho, la formación de asentamientos también se aplicó en el periodo comprendido entre fines de 1964 y la aprobación de la ley 16.640 en junio de 1967. Según recuerda Rafael Moreno, la formación de asentamientos partió 
desde el momento en que el gobierno de Frei quiso poner en marcha el proceso de Reforma Agraria, poco tiempo después de asumido. Una de las primeras iniciativas adoptadas fue la intervención del valle del Choapa, que hasta ese entonces tenía una fuerte influencia del PC, que controlaba los sindicatos campesinos existentes en esa zona (Moreno 2013: 107). De este modo se intentaba neutralizar la influencia comunista, tal como en otras zonas en años anteriores lo habían realizado sindicatos y organizaciones vinculadas a la Iglesia, la Falange y luego al PDC.

El gobierno debió enfrentar otro tipo de situaciones, como el proceso de subdivisión y de ventas ficticias que comenzaron a asumir los terratenientes de diversas provincias del país, con miras a evitar las expropiaciones contenidas en el proyecto de ley. Gómez (1985: 13) ha estimado que hasta los inicios de 1966 se subdividieron alrededor de 58 grandes haciendas, que cubrían, en conjunto, 60 mil hectáreas. Apenas se supo de esta operación de parte de los terratenientes, el gobierno decididó enviar, con urgencia, un proyecto de ley que resultó aprobado en abril de 1966 (Ley No 16.465). Por medio de esta ley quedaba extrictamente prohibido subdividir las grandes propiedades mayores a $80 \mathrm{HRB}$ sin la autorización por parte de la CORA. El gobierno, además, siguió adelante con la Reforma Agraria utilizando la legislación que había sido establecida durante el gobierno de Alessandri. Desde luego, también se hizo efectivo lo definido en la reforma constitucional de 1963, de efectuar pagos en diferido en base al avalúo fiscal de los predios que el Estado decidiera expropiar por subutilización o mal manejo productivo. Se estima que hasta antes de que fuera aprobado el proyecto de Reforma Agraria, en junio de 1967, el gobierno logró expropiar un tercio de los predios, junto con beneficiar a una proporción similar de familias en relación al total alcanzado durante todo el mandato de Frei Montalva (Barraclough y Fernández, 1974: 75; Huerta, 1989: 244). Como veremos posteriormente, tras la aprobación y puesta en vigencia de la ley 16.640, al finalizar el primer semestre de 1967 el gobierno de Frei debió enfrentar otros obstáculos que dificultaron e hicieron demasiado lento el proceso de expropiaciones.

Por otra parte, tanto en el marco de la discusión sobre la Reforma Agraria como en relación a la reforma constitucional, fue posible advertir un intenso debate sobre el problema de la propiedad. En primer lugar, los partidos de la derecha cuestionaban medidas que pudieran limitar o poner en riesgo el derecho de propiedad. Para ello se aludía a lo que la propia Constitución de 1925 garantizaba. En tal sentido, los parlamentarios de ese sector no negaban la posibilidad de que el Estado pudiera expropiar, tomando en cuenta que la propia Constitución de 1925 hacía referencia a la "función social de la propiedad", siempre y cuando tal medida no fuese incompatible con lo que estaba establecido en su artículo 10, No 10 -que garantizaba el derecho de propiedad. En segundo lugar, los sectores integristas 
de la derecha, vinculados a la revista Fiducia y al movimiento Tradición, Familia y Propiedad, negaban la potestad que podía tener el Estado para llevar a cabo medidas expropiatorias. Partían de la base de que la propiedad era condición esencial para la libertad individual, frente a lo cual se asumía una defensa irrestricta al derecho de propiedad (Cristi, 2014: 226). Además se cuestionaba la facultad interventora en relación a la propiedad, que el Estado pretendía asumir mediante las expropiaciones y medidas de tipo distributivas. Ambos sectores de la derecha se opusieron de manera categórica al proyecto de reforma constitucional y de Reforma Agraria enviado por el gobierno de Frei Montalva.

En tercer lugar, el PDC venía desde la época de la Falange formulando una propuesta que justificara la expropiación de las grandes propiedades y su distribución entre la población campesina. Sus principales ideólogos tomaban como referencia la Doctrina Social de la Iglesia, pero sobre todo la idea de bien común y de propiedad comunitaria contenida en autores como Santo Tomás y otros más contemporáneos. El uso de estas fuentes permitía realizar una interpretación diametralmente opuesta a la de los sectores integristas de las teorías del derecho natural. La Falange y luego el PDC utilizaron la revista Política y Espíritu para promover este tipo de aspectos doctrinarios que luego fueron sintetizados por el libro de Julio Silva Solar y Jacques Chonchol, El desarrollo de la nueva sociedad en América Latina ([1965]1969). Así, el PDC invertía el argumento que utilizaba la derecha para justificar la defensa de la propiedad privada. Para la derecha y los sectores integristas, la propiedad se justificaba por derecho natural, ya que para ellos se trataba de algo inherente al hombre, por el hecho de derivar de su propio esfuerzo al extraer y elaborar todo aquello que deriva de la naturaleza. Por el contrario, los ideólogos del PDC afirmaban que por derecho natural se justificaba el impulso de medidas distributivas, para de ese modo poder convertir en propietarios a quienes habiendo trabajado la tierra, no podían acceder a ella. Por último, los partidos del FRAP manifestaron un abierto apoyo al proyecto de reforma del gobierno, no obstante la postura antioligárquica que existía en amplios sectores del PS.

Con la aprobación de la ley 16.640 el gobierno va a contar con una serie de elementos jurídicos que permiten justificar la expropiación de grandes predios y su inclusión en el sector reformado. El gobierno contó con un amplio apoyo en la Cámara de Diputados, aunque tuvo que asumir varias indicaciones formuladas en el Senado. Como ya se ha dicho, la ley 16.640 establece la expropiación de todos aquellos predios mayores a $80 \mathrm{HRB}$ que estuvieran mal trabajados y explotados. Define con claridad los procedimientos de expropiación desde la notificación a sus propietarios hasta el momento en que la CORA toma posesión. De acuerdo a lo que planteaba la ley, el sector reformado se organiza mediante el sistema de asentamientos. Las atribuciones de la CORA aumentan, así como también aumenta el número de 
funcionarios y la cantidad de recursos destinados a este organismo del Estado. A diferencia de la anterior legislación, puesta en vigencia en el gobierno de Alessandri, la ley 16.640 definía como beneficiaria a población de origen campesino que se hubiese desempeñado al interior de cada predio, como ocurría con los inquilinos, medieros y otros trabajadores permanentes. Se excluía, claro está, a los trabajadores que ejercían labores de manera estacional o esporádica.

Dos meses antes de la aprobación de la ley 16.640 de Reforma Agraria había sido aprobada la ley 16.625 de Sindicalización Campesina. En el mismo primer semestre de 1967 el gobierno lograba la aprobación de dos de los proyectos más importantes en lo que concernía al problema agrario. Durante décadas ambos temas habían concitado controversia y reacción contraria de parte de la derecha, y en menor medida de colectividades como el PR. Paralelo al proceso de discusión legislativa, el gobierno había iniciado, desde los primeros meses de asumido, una activa campaña destinada a fomentar la sindicalización y la formación de dirigentes campesinos (Affonso, et al, 1970). Para este propósito el gobierno se avaló de las atribuciones que poseía el Indap. De hecho, fueron funcionarios del Indap vinculados al PDC quienes estuvieron a cargo de llevar a cabo las labores de capacitación y fomento de la organización. De manera que previo a la aprobación de la ley de Sindicalización Campesina, el PDC, a través del gobierno, había podido fomentar la constitución de sindicatos y federaciones campesinas en diversas comunas y provincias del país. Con este tipo de avances registrados por el PDC, unido al anterior proceso de formación y constitución de organizaciones por parte de la Iglesia, el gobierno lograba asegurar una importante base de apoyo para dar inicio a una fase de profundización de la Reforma Agraria. Asimismo, el PDC aseguraba su hegemonía al interior del movimiento campesino, desplazando con creces la influencia que en épocas anteriores había tenido el PC y en menor medida el PS.

\section{LA FASE DE PROFUNDIZACIÓN DE LA REFORMA AGRARIA}

Pese a los esfuerzos desplegados por el gobierno y la importante base de apoyo que poseía en el campesinado, el proceso de Reforma Agraria se vio enfrentado a una serie de situaciones de tensión, ocasionadas en parte por la institucionalidad que existía para los efectos de las expropiaciones. En parte esto también se debía a la defensa que realizaban los agricultores para evitar que sus predios fueran expropiados (Garrido, et al, 1988: 126). Así, el proceso se fue haciendo mucho más lento de lo esperado, restringiendo las posibilidades para que el gobierno pudiera alcanzar la meta de beneficiar a cerca de 100 mil familias. Por otra parte, los obstáculos también provenían de tensiones que se suscitaron al interior del partido de gobierno al surgir facciones que proponían una mayor celeridad de la Reforma 
Agraria, así como de la intensa campaña que efectuaron los terratenientes en contra del proceso.

En lo inmediato, para poder revertir los obstáculos institucionales y legales que permitían a los terratenientes utilizar una serie de subterfugios, que si bien no necesariamente impedían las expropiaciones sino que hacían más lento el proceso, surgió una iniciativa del senador Patricio Aylwin de diseñar una ley complementaria destinada a facilitar su agilización. La ley 17.280, conocida como "Ley Aylwin", puesta en vigencia a inicios de 1970, permitió agilizar el ritmo de las expropiaciones e incluso incluir inmediatamente para tales efectos a los predios que fuean objeto de "tomas" u ocupaciones por parte de sus trabajadores. Esto permitió aumentar el ritmo de las expropiaciones, en comparación a como se venían efectuando hasta ese entonces.

En términos estrictamente económicos, la Reforma Agraria permitió aumentar levemente la producción alimentaria (Barraclough y Fernández, 1974). Esto se pudo constatar hacia fines del gobierno de Frei y durante los tres años de duración del de Allende. Pero en paralelo se produjo un considerable desarrollo de la producción frutícola y forestal, así como de las actividad agroexportadora. Esto ha sido reconocido por diversos autores (Cepal, 1986; Gómez y Echenique, 1988; Tinsman, 2016). Tal fue la importancia que tuvo el desarrollo de la actividad agroexportadora que la propia revista El Campesino, pertenenciente a la SNA, publicó un reportaje titulado "Las exportaciones chilenas en los últimos años" en la edición de febrero de 1970. En base a las estadísticas del Banco Central se mostraba en dicha publicación un aumento y una diversificación de la producción de fruta fresca, seca y procesada, así como también de los mercados en Europa, Estados Unidos y Australia.

El gobierno de la UP mantuvo esta misma línea de desarrollo frutícola y agroexporador. La historiadora norteamericana Heidi Tinsman ha constatado que en 1972 se produjo un récord de exportación de toneladas de fruta en comparación con años anteriores (Tinsman, 2016: 88). El gobierno además facilitó una serie de permisos de exportación a empresarios frutícolas y, por otra parte, les ofreció a los productores vitivinícolas un régimen especial de asociación con el Estado (Del Pozo, 2014: 210). Junto a ello creó instancias para la difusión y promoción de la producción vitivinícola a visitantes extranjeros.

\subsection{Profundización de la Reforma y oposición patronal}

El aumento de las expropiaciones intensificó los conflictos que se venían generando desde que se aprueba la ley 16.640, en junio de 1967. Esto se debió al hecho de que surgieron nuevas organizaciones al alero de la SNA, aprovechando los incentivos que generaba, paradojalmente, la propia ley 16.625 de Sindicalización 
Campesina, aprobada dos meses antes. Una de las organizaciones patronales que surgió de la propia ley de Sindicalización Campesina fue la Confederación de Sindicatos de Empleadores Agrícolas 12 de junio (Consemach). Los dirigentes de esta organización reconocieron el apoyo y la asesoría legal brinada por la SNA para efectos de su constitución. Como afirmaría el propio presidente de la Consemach: "Nuestro movimiento sindical tiene una gran deuda con la SNA que, a través de su Departamento Jurídico, impulsó su creación y funcionamiento en todo el país" (Cf. Gómez, 1972: 34)5. Además de la constitución de nuevas organizaciones, que operaban en las principales comunas y provincias agrarias del país, en diversas ocasiones los terratenientes llevaron a cabo acciones en las cuales se hizo uso de la violencia. Basta citar algunos titulares y reportajes publicados por diferentes medios de prensa:

- "Gobierno exhibe debilidad ante ofensiva derechista en el campo" (Última Hora, marzo 12 de 1968).

"Chonchol denuncia complot para detener la reforma agraria" (Última Hora, septiembre 13 de 1968).

“Tensión provoca enfrentamiento campesino - patronal en Chillán” (El Siglo, octubre 17 de 1968).

"Agresión feudal a periodistas" (Última Hora, agosto 20 de 1969).

"Terretenientes de Curicó se alzan contra campesinos" (El Siglo, agosto 20 de 1969).

- "Agresores detenidos son de la organización de terratenientes" (El Siglo, agosto 22 de 1969).

- “Contra matonaje latifundista: 'El Triunfo Campesino' llama a paro nacional de advertencia” (El Siglo, agosto 23 de 1969).

- "Agricultores. La violencia de Curicó" (Ercilla, agosto 27 al 2 de septiembre de 1969).

- "Curicó: sigue la sedición derechista” (Punto Final, septiembre 9 de 1969).

- "Debilidad ante la subversión feudal (...) Atentado criminal de la derecha" (Última Hora, mayo 2 de 1970).

- "Latifundistas preparan guerra santa contra la reforma agraria" (Última Hora, mayo 2 de 1970).

- "Histórico paro campesino. 120 mil trabajadores contra la violencia latifundista" (Última Hora, mayo 12 de 1970).

5 Oszlak da cuenta de que hacia 1969, entre la Consemach, la SNA, el Consorcio Agrícola del Sur (CAS) y la Federación de Cooperativas se logró consolidar un auténtico frente de oposición a la Reforma Agraria (Oszlak, 2016: 250). 
Durante el primer semestre de 1970 recrudecieron las acciones de violencia por parte de los terratenientes. El hecho más trágico se registró el 30 de abril de 1970 en el momento en que funcionarios de la CORA intentaban tomar posesión del fundo La Piedad, ubicado en la comuna de Longaví, provincia de Linares. En el marco de esa acción fue asesinado el jefe zonal de la CORA, ingeniero agrónomo y militante del PDC, Hernán Mery (Bengoa, 2015: 304; Oszlak, 2016: 258; Avendaño, 2017: 312ss.). El hecho generó un repudio generalizado de las organizaciones campesinas, así como de los representantes de los partidos de la Unidad Popular (UP) y del PDC. Sin embargo, las acciones de violencia continuaron a tal nivel, según recuerda Moreno, que durante el mes de mayo el gobierno, a través del Ministerio del Interior, debió "presentar querellas por infracciones a la Ley de Seguridad Interior del Estado" (Moreno, 2013: 587ss.). Las acciones de violencia de parte de las organizaciones patronales y de terratenientes, quienes habían comenzado a organizar "guardias blancas", recrudecieron durante el gobierno de la UP. Asimismo continuaron con la estrategia puesta en práctica durante los últimos años del gobierno de Frei, de instigar a sus trabajadores a efectuar "tomas" y ocupaciones de los predios, y resistir en su interior, para de ese modo evitar la expropiación ${ }^{6}$.

Tabla 2. Expropiaciones efectuadas entre 1965 y 1973

\begin{tabular}{|c|c|c|c|c|c|}
\hline \multirow[t]{2}{*}{ Años } & \multirow{2}{*}{$\begin{array}{l}\mathbf{N}^{\circ} \text { de } \\
\text { predios }\end{array}$} & \multicolumn{3}{|c|}{$\begin{array}{l}\text { Superficies en hectáreas } \\
\text { (miles) }\end{array}$} & \multirow{2}{*}{$\begin{array}{l}\mathbf{N}^{o} \text { de familias } \\
\text { beneficiadas }\end{array}$} \\
\hline & & Riego & Secano & Total & \\
\hline $1965-1970$ & 1.408 & 290.6 & 3.273 .9 & 5.564 .5 & 20.976 \\
\hline $1970-1973$ & 3.628 & 394.5 & 5.190 .8 & 5.585 .3 & 33.948 \\
\hline Total & 5.036 & 685.1 & 8.464 .7 & 9.149 .8 & 54.924 \\
\hline
\end{tabular}

FUENTE: Barraclough y Fernández (1974: 75 y 85); Huerta (1989: 327).

6 Haciendo un balance del proceso reformista bajo el gobierno de Frei Montalva, Chonchol concluía que en esos años existía claramente un problema de poder, sobre todo si este se entiende "como la resistencia que, dentro de una sociedad, encuentra un proceso de reforma agraria" (Chonchol, 1971: 255-256). Bajo esta perspectiva es posible entender la razón por la cual el proceso de reforma agraria, y en particular el aumento de las expropiaciones, generaron una reacción negativa en los terratenientes y en aquellos grupos campesinos que eran controlados por ese sector. Después de todo, como explica Chonchol, no se trataba de resistencias "culturales o sociales, o también funcionales, surgidas de la manera cómo una sociedad como la nuestra reacciona frente a un proceso de cambio que pretende alterarla en uno de sus aspectos significativos" (Ibid: 256). 
Una vez en el gobierno, Allende y los representantes de la UP mantuvieron parte importante de la legislación y de las instituciones establecidas previamente. No fue necesaria una nueva reforma constitucional y para efectos de las expropiaciones se procedió con la ley 16.640, así como con la normativa adicional promovida por el senador Patricio Aylwin. El gobierno de la UP mantuvo las atribuciones definidas a la CORA y al Indap. El aspecto más innovador se expresó a través de la creación de los Centros de Reforma Agraria (Ceras) y los Centros de Producción (Cepros). Los Ceras consistían en una versión ampliada de los asentamientos. Eran formas de propiedad colectiva, de carácter transitoria, que agrupaban a varios fundos colindantes que habían sido expropiados. Los Cepros funcionaban como fincas estatales. La gestión de los Cepros dependía de técnicos y expertos definidos por la CORA y en su interior se desempeñaban trabajadores que recibían un salario por las funciones efectuadas. Los Ceras y Cepros no estaban establecidos legalmente, sino que en la práctica fueron utilizados para facilitar la gestión administrativa por parte de los organismos del Estado. El otro aspecto innovador por parte de la UP fue la formación de los Consejos Comunales Campesinos, cuya finalidad era la de cooperar con los organismos del Estado encargados de la Reforma Agraria, aunque algunos partidos de la UP y del llamado "polo revolucionario" los concibieron como "órganos de poder popular".

En términos generales, durante el gobierno de la UP se produjo un aumento considerable de las expropiaciones y de la cantidad de familias beneficiarias, en comparación con el gobierno anterior (Tabla 2). Sin embargo, cabe volver a insistir en que el gobierno de Frei debió enfrentar una serie de limitaciones legales antes de la aprobación de la ley 16.640 y antes de que se tomaran iniciativas, hacia el final de su gobierno, para facilitar los procedimientos de expropiación. Fue el gobierno de la UP el que pudo aprovechar las nuevas condiciones institucionales y legales que se generaron hacia el final del gobierno de Frei. Ahora bien, el gobierno de la UP logró aumentar las expropiaciones a tal nivel que en 1972 el latifundio prácticamente había desaparecido en todas las zonas de riego del país.

\subsection{Participación campesina}

Bajo el gobierno de Frei Montalva la Reforma Agraria se implementó fomentando al mismo tiempo la formación de sindicatos, federaciones y confederaciones campesinas. A diferencia de lo que ocurría con los gremios de terratenientes y empresarios agrícolas en general, la legislación sindical campesina tendía a generar una suerte de "divisionismo" en el movimiento campesino al fomentar la creación de federaciones y confederaciones conducidas por cada partido. De hecho, el propio PDC llegó a controlar tres confederaciones campesinas que operaban de manera autónoma. 
Tabla 3. Confederaciones sindicales campesinas, 1970-1973

\begin{tabular}{|c|c|c|c|c|c|}
\hline \multirow[t]{2}{*}{ Confederación } & \multirow{2}{*}{$\begin{array}{l}\text { Vinculación } \\
\text { partidaria }\end{array}$} & \multicolumn{2}{|l|}{1970} & \multicolumn{2}{|l|}{1973} \\
\hline & & $\mathbf{N}^{\circ}$ afiliados & $\%$ & $\mathbf{N}^{\circ}$ afiliados & $\%$ \\
\hline $\begin{array}{l}\text { Ranquil } \\
\text { Triunfo Campesino } \\
\text { Libertad } \\
\text { Sargento Candelaria } \\
\text { Unidad Obrero-Campesina } \\
\text { Provincias Agrarias Unidas }\end{array}$ & $\begin{array}{l}\text { PC-PS } \\
\text { PDC } \\
\text { PDC } \\
\text { PDC } \\
\text { MAPU } \\
\text { PN }\end{array}$ & $\begin{array}{l}35.328 \\
53.930 \\
24.854 \\
2.241 \\
--- \\
1.129\end{array}$ & $\begin{array}{l}30.10 \\
45.90 \\
21.15 \\
1.90 \\
--- \\
0.96\end{array}$ & $\begin{array}{l}96.254 \\
66.146 \\
44.260 \\
2.567 \\
14.199 \\
2.181\end{array}$ & $\begin{array}{l}43 \\
29.7 \\
19.8 \\
1.1 \\
6.3 \\
0.9\end{array}$ \\
\hline \multicolumn{2}{|l|}{ Totales } & 117.482 & 100 & 225.607 & 100 \\
\hline
\end{tabular}

FUENTE: Cereceda y Dahse (1980: 83); Bruna (1985: 112); Huerta (1989: 377).

Si se observa con detención lo que ocurre con las principales confederaciones campesinas (Tabla 3), se advierte un debilitamiento de la hegemonía que tenía el PDC durante el gobierno de Frei y un aumento de la cantidad de afiliados a la Confederación Ranquil, vinculada al PC y al PS durante el gobierno de la UP. Esto indica que la hegemonía o simplemente el predominio dentro del movimiento campesino dependió de la capacidad de control de los recursos del Estado y de la condución del proceso de Reforma Agraria.

\subsection{Las tensiones y divergencias al interior de la UP}

Desde el momento en que Allende asume el gobierno hasta fines de 1973 se produjo una división importante en la coalición oficialista, la Unidad Popular, en relación a cómo concebir y ejecutar el proceso de Reforma Agraria. Tal división fue consecuencia de la aparición de dos facciones al interior de la UP. Por un lado, aquella facción conformada por quienes asumían un compromiso incondicional con las políticas impulsadas desde el gobierno, como ocurría con el PC, el PR y un sector del PS. Por otro lado, el llamado "polo revolucionario", integrado por el sector del PS liderado por Carlos Altamirano, el Movimiento de Acción Popular Unitaria (MAPU), la Izquierda Cristiana (IC) y el Movimiento de Izquierda Revolucionaria (MIR). Como es sabido, el MIR no integraba la UP, pero estableció una plataforma común con el PS liderado por Carlos Altamirano.

Desde el punto de vista programático, el hito más significativo de presión por la profundización de la Reforma Agraria de parte del "polo revolucionario" se produjo con la "Declaración de Linares", formulada a fines de 1971. La "Declaración" fue dada conocer a través de un extenso documento que fue difundido por la revista 
Punto Final y El Rebelde, principalmente. La "Declaración” pretendía representar un punto de inflexión, o bien de ruptura, frente al carácter gradual con que se había conducido el proceso de Reforma Agraria, y sobre todo promover una nueva legislación distinta a la promulgada por el gobierno de Frei Montalva ${ }^{7}$. Los contenidos de esta nueva normativa debían apuntar a sustituir muchos aspectos de lo definido en la ley 16.640 aprobada en junio de 1967. Se consideraba que esa legislación mantenía beneficios para los latifundistas, como por ejemplo el derecho a "reserva", es decir, poder escoger las tierras no expropiadas dentro de las más fértiles y productivas. Además, se planteaba reducir el requisito de expropiación de 80 a 40 HRB para de ese modo debilitar a la "burguesía agraria" que se estaba constituyendo como consecuencia de la reforma. A su vez, consideraba que las expropiaciones debían ser realizadas "a puertas cerradas"; es decir, no sólo debía ser expropiada la tierra sino también los animales, la maquinaria, las herramientas y todo lo producido en su interior. Por último, se pretendía hacer de los Consejos Campesinos órganos de poder en los cuales los trabajadores pudieran participar en la toma de decisiones de todo aquello relacionado con la política agraria.

Por su parte, el PC apoyaba incondicionalmente la política agraria definida en el programa de gobierno de la UP. Con anterioridad había rechazado de manera categórica los planteamientos del "polo revolucionario" de la izquierda. Frente a la propuesta de expropiar más allá de lo establecido por la ley, incluyendo el ganado o la maquinaria, el Secretario General del PC, Luis Corvalán, descartaba completamente ese tipo de iniciativas. La única alternativa al respecto podría ser "que el Estado pueda resolver el problema sobre la base de la importación de tractores, equipos agrícolas, etc." (Principios. No 137, enero-febrero de 1971: 79). Por último, Corvalán se oponía rotundamente al impulso de "tomas" u ocupaciones ilegales de los predios agrícolas, debido a que según él no se justificaban en el marco de lo que consideraba un gobierno popular.

7 "Plantea la creación de una nueva Ley de Reforma Agraria. Niega el derecho reserva y el pago de las indemnizaciones. Impulsa los Consejos Campesinos como organismos de poder de los campesinos, para que desde allí ellos tengan participación en la política agraria y tomen decisiones que sirvan a sus intereses de clase" (El Rebelde, enero 7 de 1972: s/p). Más adelante, el Movimiento Campesino Revolucionario (MCR), perteneciente al MIR, sostendría: "que cada vez se hace más urgente terminar con la Reforma Agraria que se planteó durante el gobierno de Alessandri y se puso en práctica en el gobierno de Frei (....) El MCR ha entendido incansablemente que sólo una ley revolucionaria que interprete fielmente los intereses de los campesinos podrá acabar con la explotación en el campo" ("Linares: seguir en la ofensiva es impulsar la revolución”. El Rebelde, enero 19 de 1972: s/p.). 


\section{CONCLUSIONES}

De acuerdo a lo que ha sido expuesto anteriormente, en Chile la Reforma Agraria fue el resultado de un proceso de transformación gradual que comenzó a partir de las iniciativas emprendidas por Jorge Alessandri en 1962. Es también el resultado de diagnósticos y propuestas programáticas que el PS, el PC y el PDC formularon en las décadas anteriores, al plantear la necesidad de revertir los problemas de la agricultura y que aquejaban al campesinado y a los trabajadores agrícolas en general, mediante la expropiación del gran latifundio y la posterior distribución de tierra en esos segmentos de la población rural. No obstante, el carácter gradual de la transformación que conlleva la Reforma Agraria permite identificar distintas concepciones y formas en que fue asumida por los gobiernos sucesivos. Desde luego, la aceleración del proceso de Reforma coincide con el periodo de mayor tendencia hacia la polarización y la confrontación ideológica y política, como ocurre a partir de 1967 y sobre todo en el periodo comprendido entre 1970 y 1973.

Que la Reforma Agraria se haya expresado al menos de tres maneras es consecuencia de las distintas concepciones del cambio estructural que primaba entre los partidos y organizaciones gremiales existentes. No hubo sólo posiciones dicotómicas en el comportamiento de los partidos, ni en la forma en que fue asumido el proceso reformista. Una clara demostración se puede reconocer en los años del gobierno de la UP, donde entre los partidos de izquierda se configuraron dos diagnósticos e interpretaciones distintas de la realidad social del sector rural, así como dos formas de concebir la Reforma Agraria.

Uno de los rasgos más sobresalientes que tuvo la Reforma Agraria desde que se implementó de manera efectiva a partir de 1964, fue su carácter multidimensional. Es decir, lo que estuvo en juego no fue sólo la expropiación y asignación de tierra, ni tampoco la necesidad de estimular unidades de producción más eficientes. También estuvo en juego el problema del agua y de asegurar su dominio público por parte del Estado, el fomento productivo, el acceso al crédito, la innovación técnica, la participación y organización campesina. Esto explica por qué la Reforma Agraria se acompañó de una institucionalidad ad-hoc compuesta por una serie de organismos públicos especializados, y por otros que reforzaron el proceso en el ámbito de la formación y la capacitación de dirigentes campesinos. 


\section{REFERENCIAS}

Affonso, Almino, Sergio Gómez, Emilio Klein y Pablo Ramírez. Movimiento campesino chileno. Vol. I. Santiago: ICIRA, 1970.

Ahumada, Jorge. En vez de la miseria. Santiago: Ediciones Bat, 1990. 10ª edición (1ª edición 1958).

Albertus, Michael. Autocracy and Redistribution. The Politics of Land Reform. Cambridge: Cambridge University Press, 2015.

Avendaño, Octavio. Lospartidos frente a la cuestión agraria en Chile, 1946-1973. Representación de intereses, gradualismo y cambio estructural. Santiago: Lom Ediciones, 2017.

Avendaño, Octavio. "La reforma agraria durante el gobierno del presidente Eduardo Frei Montalva: un cambio estructural y la incorporación del campesinado". En C. Huneeus y J. Couso (eds.): Eduardo Frei Montalva: un gobierno reformista. A 50 años de la "Revolución en Libertad". Santiago: Editorial Universitaria, 2016, pp. 159-195.

Avendaño, Octavio y María Cristina Escudero. "Elitismo y poder gremial en la Sociedad Nacional de Agricultura (SNA)". Revista de Ciencias Sociales No 20, septiembre-diciembre, 2016, pp. 37-74.

Barraclough, Solon y José Antonio Fernández. Diagnóstico de la reforma agraria chilena. México: Siglo XXI Editores, 1974.

Bengoa, José. Historia rural de Chile central. Tomo II. Crisis y ruptura del poder hacendal. Santiago: Lom Ediciones, 2015.

Bengoa, José. Reforma Agraria y revuelta campesina. Santiago: Lom Ediciones, 2016.

Brahm, Enrique. Propiedad sin libertad: Chile 1925-1973. Aspectos relevantes en el avance de la legislación socializadora. Santiago: Universidad de los Andes. Colección Jurídica, 1999.

Bruna, Susana. "Chile: las luchas campesinas en el siglo XX”. En P. González Casanova (coord.). Historia politica de los campesinos latinoamericanos. Vol. 4. México: Siglo XXI Editores, 1985.

Cardemil, Alberto. El buaso chileno. Santiago: Editorial Andrés Bello, 2000.

CEPAL. El desarrollo fruticola y forestal y sus derivaciones sociales. Santiago: Naciones Unidas-CEPAL, 1986.

Cereceda, Luz y Fernando Dahse. Dos décadas de cambio en el agro chileno. Santiago: Instituto de Sociología, Pontificia Universidad Católica, 1980. 
Chonchol, Jacques. Sistemas agrarios en América Latina. De la etapa prehispánica a la modernización conservadora. México-Santiago: Fondo de Cultura Económica, 1994.

Chonchol, Jacques. "Poder y reforma agraria en la experiencia chilena". En A. Pinto, S. Aranda, A. Martínez, O. Caputo, R. Pizarro, E. Faletto, E. Ruiz, J. Chonchol, V. Brodersohn, T. Vasconi, I, Reca y A. Dorfman. México-Santiago: Siglo XXI Editores-Editorial Universitaria, 1971, pp. 255-321.

Correa, Sofía. Con las riendas del poder. La derecha chilena en el siglo XX, Santiago: Editorial Sudamericana, 2004.

Cousiño V., Ángela y María Angélica Ovalle. Reforma Agraria chilena. Testimonios de sus protagonistas. Santiago: Editorial Memoriter, 2013.

Cristi, Renato. "La síntesis conservadora/neoliberal de Jaime Guzmán: la subsidiaridad como principio articulador". En R. Cristi y P. Ruiz-Tagle. El constitucionalismo del miedo. Propiedad, bien común y poder constituyente. Santiago: Lom Ediciones, 2014, pp. 209-229.

Del Pozo, José. Historia del vino chileno. Desde la época colonial hasta hoy. Santiago: Lom Ediciones, 2014.

Echenique, Jorge. "Las expropiaciones y la organización de los asentamientos en el período 1965-70”. En D. Alaluf, et al. Reforma Agraria chilena: Seis ensayos de interpretación. Santiago: Icira, 1972, pp. 93-110.

Fontaine, Arturo. La tierra y el poder. Reforma Agraria en Chile (1964-1973). Santiago: Zig-Zag, 2001.

Frank, Andre G. “Tipos de reformas agrarias". En O. Delgado (ed.). Reformas Agrarias en América Latina. México: Fondo de Cultura Económica, 1965, pp. 184-188.

Gamboa, Ricardo. "Reformando reglas electorales: La cédula única y los pactos electorales en Chile (1958-1962)". Revista de Ciencia Política. Vol. 31. No 2, 2011, pp. 159-186.

García, Antonio. Reforma Agraria y economía empresarial en América Latina. Santiago: Editorial Universitaria, 1967.

Garrido, José, Cristián Guerrero y María Soledad Valdés. Historia de la Reforma Agraria en Chile. Santiago: Editorial Universitaria, 1988.

Goic, Pedro y Enrique Sanhueza. Un camino en la dignificación del campesino. Santiago: Colorama S. A., 1992. 
Gómez, Sergio. “Tenencia de la tierra, Chile 1965-1985”. Documento de Trabajo N 286. Santiago: Flacso, 1986.

Gómez, Sergio. Los empresarios agrícolas. Santiago: Icira, 1972.

Gómez, Sergio y Jorge Echenique. La agricultura chilena. Las dos caras de la modernización. Santiago: Flacso-Agraria, 1988.

Gómez M., Ariel. El Partido Radical durante el gobierno de Jorge Alessandri, 1958-1964. Tesis para optar al grado de Licenciado en Ciencias Jurídicas y Sociales. Santiago: Facultad de Derecho, Universidad de Chile, 2003.

Huerta, María Antonieta. Otro agro para Chile. La bistoria de la Reforma Agraria en el proceso socialy politico. Santiago: Cisec-Cesoc, 1989.

Lapp, Nancy D. Landing Representation and Land Reform in Latin America Votes. Nueva York: Palgrave MacMillan, 2004.

Loveman, Brian. Struggle in the Countryside. Politics and Rural Labor in Chile, 1919-1973. Bloomington: Indiana University Press, 1976.

Mattelart, Armand. "Apuntes sobre el concepto de reversibilidad de un proceso de reforma agraria". En D. Alaluf, et al. Reforma Agraria chilena: Seis ensayos de interpretación. Santiago: Icira, 1972, pp. 69-92.

McCoy, Terry L. "The Politic of Structural Change in Latin America: The case of Agrarian Reform in Chile". Working Paper No 37. Ohio State University, 1969.

Moreno, Rafael. Sin reforma agraria no habria sido posible. Memorias de la Reforma Agraria chilena 1958-1970. Santiago: Ediciones Copygraph, 2013.

Oszlak, Oscar. La trama oculta del poder. Reforma agraria y comportamiento político de los terratenientes chienos, 1958-1973. Santiago: Lom Ediciones, 2016.

Pinto, Aníbal. Chile, un caso de desarrollo frustrado. Santiago: Editorial Universitaria, 1958.

Silva, Julio y Jacques Chonchol. El desarrollo de la nueva sociedad en América Latina. Santiago: Editorial Universitaria. $2^{a}$ edición, 1969 (1ª edición 1965).

Urzúa, Raúl. "Notas acerca de la estructura de poder en Chile". En R. Atria, A. Bardón, S. Molina, E. Ortega y R. Urzúa. Hacia un nuevo diagnóstico de Chile. Santiago: Editorial del Pacifico, 1973, pp. 101-140.

Valdés, Alberto y William Foster. La Reforma Agraria en Chile. Historia, efectos y lecciones. Santiago: Ediciones Universidad Católica de Chile, 2015. 